Bio - grafía. Escritos sobre la Biología y su Enseñanza. ISSN 2027-1034

Edición Extraordinaria. p.p. 839 - 847

Memorias del IX Encuentro Nacional de Experiencias en Enseñanza de la Biología y la Educación Ambiental. IV Congreso Nacional de Investigación en Enseñanza de la Biología.

\title{
REPRESENT ACIONES SOCIALES SOBRE EL CAMBIO CLIMÁTICO Y SUS EFECTOS EN ADOLESCENTES DE INSTITUCIONES OFICIALES Y PRIVADAS DE MONTERÍA - CÓRDOBA
}

\section{SOCIAL REPRESENT ATIONS ON CLIMATE CHANGE AND ITS EFFECTS ON ADOLESCENTS FROM OFFICIAL AND PRIVATE INSTITUTIONS IN MONTERÍA - CÓRDOBA}

\author{
Francisco Portillo Páez ${ }^{1}$ \\ Miriam Vega Vega ${ }^{2}$ \\ Edith Cadavid Velásquez ${ }^{3}$ \\ Nabi Pérez Vásquez ${ }^{4}$
}

\section{RESUMEN}

Se compararon las representaciones sociales de adolescentes sobre el cambio climático, causas y consecuencias de cuatro instituciones educativas de Montería, dos oficiales: Nacional José María Córdoba y Mercedes Abrego y dos privadas: Colegio La Salle y Windsor Royal School, grupo focal con edades entre los 12 y 16 años. Caracterizando sus saberes cotidianos y contrastándolos con información científica. Con un enfoque cualitativo mediante estudio de caso, se aplicaron entrevistas a profundidad analizadas mediante Atlas ti. Los resultados muestran que los adolescentes de instituciones públicas tienen concepciones erróneas sobre cambio climático, afiman no consultar sobre el tema y visitan pocos sitios de debate. Los estudiantes de instituciones privadas, muestran mayor apropiación del tema, relacionado con el acceso a información, asocian el concepto de cambio climático con cambios en el clima, sin embargo señalan erróneamente que es estacionario. Respecto a la adaptación no plantean respuestas acertadas, asocian el fenómeno a variabilidad climática y que el ser humano puede adaptarse al estar en diferentes climas, respuestas recurrentes en los adolescentes entrevistados, por tanto, se debe fortalecer un proceso crítico, reflexivo y participativo para el generar acciones individuales y colectivas de mitigación y adaptación a través de talleres pedagógicos de formación.

\footnotetext{
${ }^{1}$ Estudiante Licenciatura en Ciencias Naturales y Educación Ambiental. Universidad de Córdoba. franporti1991@hotmail.com

2 Estudiante Licenciatura en Ciencias Naturales y Educación Ambiental. Universidad de Córdoba. Miriam.m.vega@gmail.com

${ }^{3}$ Docente Universidad de Córdoba. Licenciada en Biología y Química, Magister en Ciencias Ambientales.ejcajave@yahoo.com

${ }^{4}$ Docente Universidad de Córdoba. Licenciada en Biología y Química, Magister en Ciencias Ambientales. Estudiante de Doctorado en Educación y Cultura Ambiental. ndperezvasquez@correo.unicordoba.edu.co
} 
Bio - grafía. Escritos sobre la Biología y su Enseñanza. ISSN 2027-1034

Edición Extraordinaria. p.p. 839 - 847

Memorias del IX Encuentro Nacional de Experiencias en Enseñanza de la Biología y la

Educación Ambiental. IV Congreso Nacional de Investigación en Enseñanza de la Biología.

PALABRAS CLAVE: cambio climático, representaciones sociales, adolescentes, adaptación y mitigación.

ABSTRACT.

The social representations of adolescents on climate change, causes and consequences of four educational institutions in Monteria were compared, two officers: Nacional José María Córdoba and Mercedes Abrego and two private ones: College La Salle and Windsor Royal School, a focal group with ages between 12 and 16 years. Characterizing their everyday knowledge and contrasting it with scientific information. With a qualitative approach by means of a case study, depth interviews were analyzed using Atlas ti. The results show that adolescents from public institutions have misconceptions about climate change, say they do not consult about the topic and visit few discussion sites. The students of private institutions, show greater appropriation of the subject, related to the access to information, associate the concept of climate change with changes in the climate, but erroneously indicate that it is stationary. Regarding adaptation do not present correct answers, they associate the phenomenon with climatic variability and that the human being can adapt to being in different climates, recurrent responses in adolescents interviewed, therefore, a critical, reflexive and participatory process for the Generate individual and collective actions of mitigation and adaptation through pedagogical training workshops.

KEYWORDS: climate change, social representations, adolescents, adaptation and mitigation.

\section{INTRODUCCIÓN.}

El cambio climático (CC) es un hito en la historia natural del mundo sobre el que existen desacuerdos en relación a la gravedad, acciones para afrontarlo y costos de mitigación (Rozell, 2017), algunos afirman no se requiere medidas para sobrellevarlo y otros las consideran necesarias (Bushell, Buisson, Workman, \& Colley, 2017). Se concibe como problema de generaciones futuras y de riesgo inminente en la sociedad actual, debido a sus manifestaciones. Hay polémica sobre el fenómeno y esta se relaciona más con los mecanismos utilizados para comunicarlo, que con sus evidencias científicas, medios de comunicación poco rigurosos han contribuido a crear una imagen catastrófica (Camarasa Belmonte \& Moreno Sanz, 1994), con información deficiente, introduciendo falsas atribuciones y erróneas interpretaciones (Vide, 2008). Si bien la alfabetización científica y el razonamiento técnico no predice la percepción de los riegos (Leombruni, 2015), se requieren procesos de adaptación que ajusten alternativas, realizar estudios sobre la base social del problema, preguntándose el porqué de las actitudes de las personas y como cambiarlas (Kaltenborn, Krange, \& Tangeland, 2017).

Es importante reconocer que la concepción de las problemáticas ambientales proviene de las percepción empírica, lo comunicado por científicos y académicos y la herencia cultural como conocimiento arraigado en la forma de actuar y pensar de la humanidad, por ello es necesaria la participación de la sociedad en el análisis de amenazas del cambio climático, 
Bio - grafía. Escritos sobre la Biología y su Enseñanza. ISSN 2027-1034

Edición Extraordinaria. p.p. 839 - 847

Memorias del IX Encuentro Nacional de Experiencias en Enseñanza de la Biología y la

Educación Ambiental. IV Congreso Nacional de Investigación en Enseñanza de la Biología.

para que se conozcan y entiendan los riesgos y oportunidades para afrontarlos (Chambers, 2009), desarrollando compromisos para implementar soluciones concretas. El Panel Intergubernamental de Cambio Climático (IPCC) contienen datos científicos útiles y efectivos los cuales deben ser integrados a procesos de producción a través de métodos participativos de conocimiento y dialogo (Pearce, Brown, Nerlich, \& Koteyko, 2015), siendo importante conocer los saberes de las sociedades, es decir sus representaciones sociales (RS), como sistemas cognitivos con lenguaje y lógica, en este sentido, (Moscovici, 1984) plantea que las RS no son simples opiniones, imágenes o actitudes, sino teorías de conocimiento destinadas a interpretar la realidad, relacionadas con simbolizaciones de experiencia social, elaboradas por los sujetos. En general, las representaciones sociales, las percepciones públicas, las dinámicas sociales y las evidencias científicas son elementos que construyen la historia del cambio climático (Evans \& Steven, 2007); conocimiento necesario para plantear estrategias de intervención pedagógica de adaptación, siendo el interés el punto de entrada que permite contextualizar los efectos del fenómeno, de manera que tenga sentido para ellos, apoyados en la premisa de que los valores moldeados a través de la vida están influenciados por la socialización y la cultura temprana, que a su vez permean actitudes hacia el CC (Kaltenborn et al., 2017).

Hay que destacar que para enfrentar el cambio climático, se requieren acciones de adaptación, en las que son necesarias la educación y apropiación de información científica desde las instituciones educativas, en ellas existen representaciones idóneas, equivocas, imaginarias e incluso nulas con respecto al problema, lo que nos llevó al interés de evaluar las representaciones sociales de adolescentes del contexto local a fin de conocer la probabilidad de adoptar conductas y apoyar acciones para mitigar el riesgo asociados al fenómeno, lográndose identificar el nivel de sensibilidad, información y comprensión del conocimiento que sobre éste tenían, para fortalecer la participación de los estudiantes en acciones que conduzcan a contrarrestar el fenómeno, propiciando mecanismos culturales hacia la reducción de la vulnerabilidad social. Para adaptarse al Cambio Climático se requiere que la sociedad lo conozca, comprenda y evalué las estrategias para implementarlas, el estudio sobre la percepción y el nivel de conciencia ciudadana al CC busca identificar qué puede ser hecho, en qué forma y por quién (Retamal, Rojas, \& Parra, 2011), más aun cuando el IPCC propone investigar la actitud y posición de la población frente al fenómeno a fin de formular estrategias educativas y de comunicación (IPCC, 2014), en este sentido, las representación sociales de los adolescentes sirven para orientar la acción (Flores, 2015) que se debe seguir para afrontar dicho problema.

\section{METODOLOGÍA.}

La investigación se situó en el paradigma cualitativo, utilizando técnicas flexibles y abiertas (Bisquera, 2004) para comprender e interpretar el fenómeno (Hernández, Fernández, \& Baptista, 2010). Mediante estudio de Caso, buscando la comprensión de complejas relaciones (Stake, 2007), método particularista, descriptivo, heurístico e inductivo (Muñoz \& Muñoz, 2001; Serrano, 1994), analizando problemas de la practica 
Bio - grafía. Escritos sobre la Biología y su Enseñanza. ISSN 2027-1034

Edición Extraordinaria. p.p. 839 - 847

Memorias del IX Encuentro Nacional de Experiencias en Enseñanza de la Biología y la

Educación Ambiental. IV Congreso Nacional de Investigación en Enseñanza de la Biología.

social y educativa (Merrian,1988); siguiendo la estructura de Simons, descripción, análisis e interpretación (Simons, 2011). La población escogida adolescentes de instituciones oficiales Mercedes Ábrego, Nacional José María Córdoba y privadas Colegio la Salle y Windsor Royal School de Montería. Se determinó una muestra aleatoria por conveniencia de 15 estudiantes por institución, para un total de 60 adolescentes, a quienes se les realizó entrevista a profundidad como instrumentos de recolección de información (Abric, 1994; Correa, 2015; Flores, 2015; Moscovici, 1984; Stake, 2007), del mismo modo se realizaron observaciones directas, analizando las dimensiones de las representaciones sociales, campo de representación y actitudes. Se utilizó el software Atlas ti para el análisis de la información, indicado porque integra, una red estructural compleja, las realidades poliédricas que presentan procesos psicológicos, sociales, antropológicos y sociopolíticos (Martínez, 2006).

\section{RESULTADOS Y DISCUSIÓN.}

Se identificaron las representaciones sociales de adolescentes mediante las entrevistas a profundidad y estas fueron analizadas a través del Software Atlas ti, se concretaron las categorías de conocimiento sobre cambio climático, causas, consecuencias mitigación, adaptación y sitios de debate.

Cambio climático; el análisis de Atlas ti permitió correlacionar ésta categoría a las de desconocimiento y sitios de debate (Figura $1 \mathrm{~A}$ y $1 \mathrm{~B}$ ), fundamentando en la desvaloración actitudinal respecto a la problemática. Los adolescentes de instituciones públicas no muestran argumentos válidos sobre cambio climático, en sus voces se establecen concepciones sin fundamentación científica, "es como estar en una parte fría y pasar a otra caliente, porque si yo estoy acostumbrado a una parte fría y me voy para la caliente entonces mi cuerpo puede sufrir algunas consecuencias, entonces eso para mí es cambio climático" (Estudiante del Nacional José María Córdoba, 13 años). Además manifestaron no consultar sobre el tema. Las voces, de estudiantes de instituciones privadas, evidencia acceso a la información, asocian el concepto de CC con cambios en el clima, "El cambio del clima en algunos lugares del país o del mundo" (Estudiante de La Salle, 14 años), sin embargo plantean que es un problema estacionario, lo que permite afirmar que no hay dominio del concepto si bien tienen asequibilidad a la información, carecen de interés por consultar sobre el problema; (González \& Maldonado, 2014) plantearon que para entender el CC hay que tener en cuenta actitudes y conocimientos, en tanto que, la falta de interés en el tema conlleva a mayor desconocimiento, por ello hay que motivarlos e implementar estrategias formativas para la apropiación de información desde las instituciones educativas. 
Bio - grafía. Escritos sobre la Biología y su Enseñanza. ISSN 2027-1034

Edición Extraordinaria. p.p. 839 - 847

Memorias del IX Encuentro Nacional de Experiencias en Enseñanza de la Biología y la

Educación Ambiental. IV Congreso Nacional de Investigación en Enseñanza de la Biología.

Causas del cambio climático: Para esta categoría, se resalta el conocimiento vivencial de los adolescentes de instituciones públicas (figura $1 \mathrm{~A}$ ) debido a que están inmersos en algunas de sus manifestaciones, en sus voces, relacionan el fenómeno con algunas causas como la falta de cultura ambiental, quema de basura, debido a la carencia de servicios de recolección, exceso de $\mathrm{CO}_{2}$ por automóviles y fábricas y tala de árboles, mencionan situaciones vividas, dejando de lado otros factores "La suciedad, porque estamos tirando mucha basura y eso contamina el ambiente" (Estudiante del Nacional José María Córdoba, 12 años). Según (Alvear, Ceballos, \& Bolaños, 2015) la responsabilidad que tienen diferentes actores sociales sobre el cambio climático se relaciona con su vivencia cotidiana, en su estudio como en el nuestro, actividades como la ganadería y la agricultura con gran aporte al Cambio Climático no fueron consideradas por los entrevistados. Los adolescentes de instituciones privadas, debido al contexto socio-económico señalan, en sus voces que no evidencian en su entorno causas del fenómeno, asocian el tema con contaminación ambiental, por emisiones de $\mathrm{CO}_{2}$ de automóviles y fábricas "las causas de cambio climático son la ruptura de capa de ozono lo que contaminación del mundo" (Estudiante del Windsor, 12 años.); de acuerdo con (González \& Maldonado, 2014) se presentan confusiones sobre las causas del CC y otros problemas ambientale

Consecuencias del cambio climático: los adolescentes de instituciones públicas,
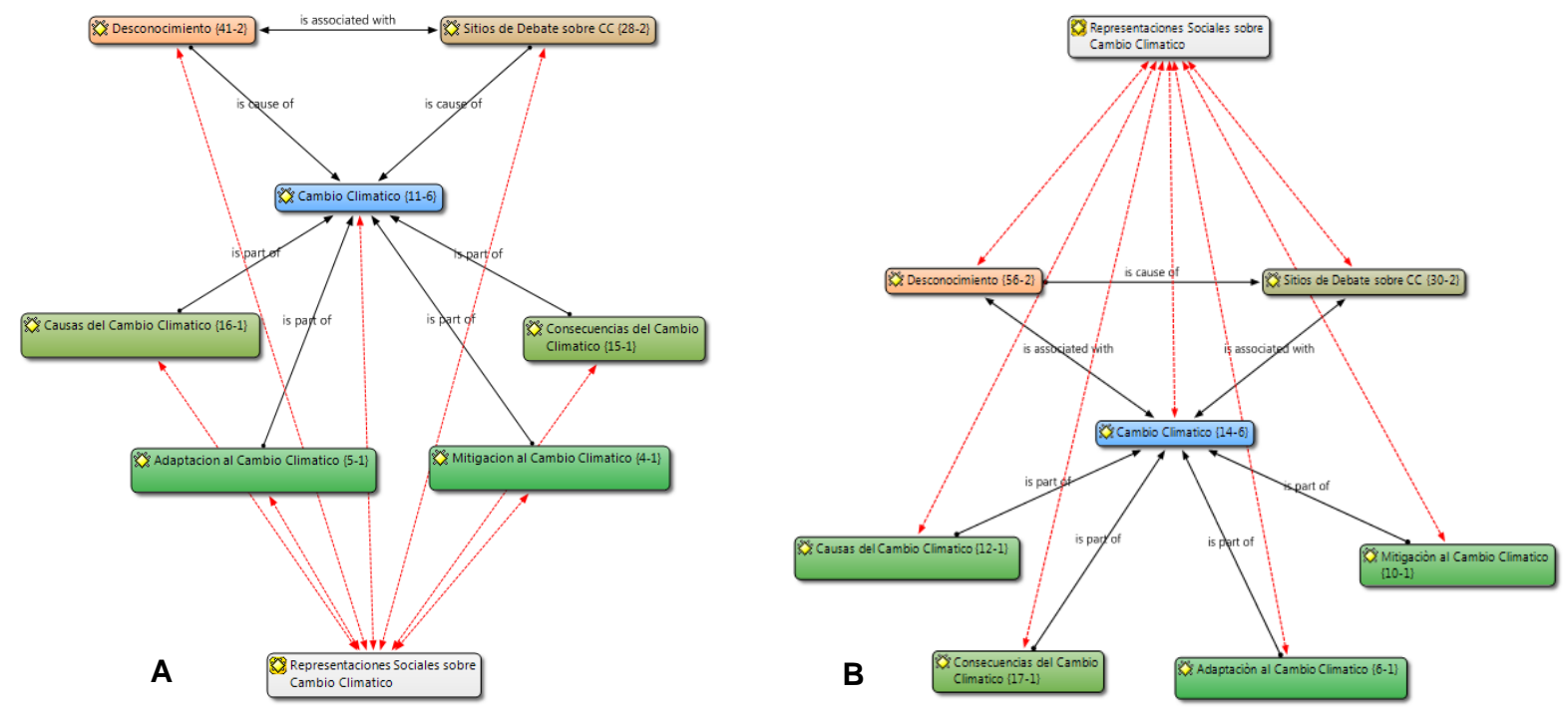

Figura 1. Figura 1. Representaciones sociales sobre cambio climático de estudiantes de Instituciones Educativas. 1A. Públicas, Nacional José María Córdoba y Mercedes Ábrego. 1B. Privadas, La Salle y Windsor Royal School

correlacionan causas con consecuencias, debido a que estos asumen la variabilidad en el clima producto de acciones negativas del ser humano, tala de árboles, quema de basuras, contaminación de ríos, reflejado en desastres naturales, enfermedades, aumento de temperatura, siendo el argumento al que atribuyen como consecuencia del CC, desconociendo otras que por trascendencia dejan huellas ambientales irreversibles; "el problema del cambio climático se están dando gracias a la contaminación, muchos carros, 
Bio - grafía. Escritos sobre la Biología y su Enseñanza. ISSN 2027-1034

\title{
Edición Extraordinaria. p.p. 839 - 847
}

\author{
Memorias del IX Encuentro Nacional de Experiencias en Enseñanza de la Biología y la \\ Educación Ambiental. IV Congreso Nacional de Investigación en Enseñanza de la \\ Biología.
}

quema de basura un ejemplo seria en Mocoa, los niños de Mocoa que sufrieron una inundación y pienso que eso fue por el descuido de la gente" (Estudiante de Mercedes Abrego, 13 años). (Correa, 2015), afirma que los estudiantes desconocen los efectos de los gases de efecto invernadero y no comprenden las consecuencias del cambio climático. Los adolescentes de instituciones privadas, dan respuestas globales frente a las consecuencias del CC "entre las consecuencias del fenómeno están el derretimiento de los polos, también que haya mucho calor o que se formen los tornados, terremotos, tsunamis" (Estudiante del Windsor, 13 años.); (Flores, 2015) plantea que los medios de comunicación tienen relevancia en la conformación de las representaciones sociales sobre el cc, entre la información transmitida en los últimos años se habla del deshielo de glaciares, información reconocida por los estudiantes en un 80\%, como consecuencia del cambio climático, también manifiestan la destrucción de la capa de ozono, de lo que se infiere que existe más acceso de información y por tanto mayor conocimiento.

Adaptación al Cambio Climático: esta es una categoría, que tuvo pocas respuestas, asocian el fenómeno a variabilidad climática y que el ser humano puede adaptarse al estar en diferentes climas, cabe anotar que estas respuestas fueron recurrentes en la mayoría de los adolescentes entrevistados (instituciones públicas y privadas), "si, si somos seres humanos es natural adaptarnos" (Estudiante de La Salle, 12 años.) Lo que reafirma la necesidad de tomar medidas educativas, los adolescentes tienen poco conocimiento sobre estrategias de adaptación al fenómeno, en sus voces, lo asocian a pisos térmicos, manifestando que se puede pasar de un clima cálido a uno frio y que eso no requiere una acción por parte de ellos, infiriéndose que frente al conocimiento científico tienen ideas erróneas, lo que impiden que se apropien de un conocimiento adecuado para atender las dificultades del cambio climático; (Moraes \& Montes, 2011) consideran que las estrategias de mitigación y adaptación tienen influencia significativa en el desarrollo de programas políticos así como en acciones individuales relacionadas al consumo energético.

Mitigación al cambio climático: Con relación a esta categoría se destaca que las acciones realizadas por los adolescentes se queda en conciencia ambiental, en sus voces muestran poco interés al respecto, y plantean acciones como recoger basura, sembrar árboles, reducir el consumo de agua, quedándose en acciones primarias, que no tienen una trascendencia en largos periodos de tiempo, lo que es fundamental para reducir los efectos del cambio climático. "Prácticamente recojo cualquier basurita que hay por allí, reciclo y algunas veces siembro un árbol" (Estudiante de Mercedes Abrego, 12 años) Los adolescentes de las instituciones privadas, en sus voces muestran la mitigación solo como acciones que ayudan a mejorar el ambiente, teniendo en cuenta el contexto en el que viven, manifiestan, reducir el uso de automóviles y utilizar menos plástico y reciclar "Cuando voy en el carro no boto la basura por la ventana, trato de no andar mucho en moto o carro prefiero ir en bicicleta para no contaminar el medio ambiente" (Estudiante de La Salle, 12 años.) 
Bio - grafía. Escritos sobre la Biología y su Enseñanza. ISSN 2027-1034

Edición Extraordinaria. p.p. 839 - 847

Memorias del IX Encuentro Nacional de Experiencias en Enseñanza de la Biología y la

Educación Ambiental. IV Congreso Nacional de Investigación en Enseñanza de la Biología.

Sitios de debate: El acceso a la información por parte de los adolescentes de las instituciones públicas es regular, las fuentes consultadas no son veraces, lo que crea representaciones sociales errónea acerca del cambio climático, mencionan fuentes como Facebook, televisión y vecinos, que en muchos casos no cuentan con soporte científico sobre la problemática, " he escuchado hablar del cambio climático a través del noticiero, y de mis vecinos" (Estudiante de Mercedes Àbrego, 13 años) siendo limitada su formación, no valoran el conocimiento científico, resaltando el efecto que causan algunos medios de comunicación con informaciones poco acertadas sobre CC, conduciendo a representaciones incorrecta para afrontar este tema; (Camarasa Belmonte \& Moreno Sanz, 1994), señalaron que medios de comunicación presentan información rápida y deficiente, introduciendo falsas atribuciones y erróneas interpretaciones. Los adolescentes de instituciones privadas, utilizan diversos sitios de debate, dado al acceso a información por su contexto socio- económico, sin embargo, es manejada superficialmente, aunque tengan acercamiento, el tema no les genera interés, por ello el desconocimiento no está asociado a la accesibilidad de información para este caso, sino a la falta de interés. "A través de las clases de ciencias naturales, biología, por mi colegio, mis padres, televisión, internet, pero no consulto sobre el tema" (Estudiante La Salle, 13 años.). Cabe resaltar que se debe generar una disposición para un mejor conocimiento acerca del "cambio climático".

\section{CONCLUSIONES.}

Los adolescentes manejan concepciones erróneas sobre el cambio climático, muestran desinterés por el tema y sus sitios de debate no son eficientes y eficaces, por lo que se requieren estrategias de formación sobre el fenómeno para la adopción de medidas de mitigación y adaptación, lo que implica cambios en estilos de vida, modificar comportamientos, con actitudes y patrones de consumo, propiciando su disposición a llevarlas a cabo, siendo consciente de las implicaciones del problema lográndose especial atención en aspectos de vulnerabilidad social.

\section{BIBLIOGRAFÍA.}

Abric, J.-C. (1994). L'organisation interne des représentations sociales: système central et système périphérique, In, C., Guimelli. Structure et transformation des représentations sociales.

Alvear, N., Ceballos, V., \& Bolaños, M. (2015). Los jóvenes de la ciudad de Popayán frente al cambio climático. Un estudio desde las representaciones sociales. Revista Ingenierías Universidad de Medellín, 14(26).

Bisquera, R. (2004). Metodología de la investigación educativa (Vol. 1): Editorial La Muralla.

Bushell, S., Buisson, G. S., Workman, M., \& Colley, T. (2017). Strategic narratives in climate change: Towards a unifying narrative to address the action gap on climate change. 
Bio - grafía. Escritos sobre la Biología y su Enseñanza. ISSN 2027-1034

Edición Extraordinaria. p.p. 839 - 847

Memorias del IX Encuentro Nacional de Experiencias en Enseñanza de la Biología y la Educación Ambiental. IV Congreso Nacional de Investigación en Enseñanza de la Biología.

Energy Research \& Social Science, 28, 39-49. doi: http://doi.org/10.1016/j.erss.2017.04.001

Camarasa Belmonte, A. M., \& Moreno Sanz, F. (1994). Algunas reflexiones sobre la percepción del cambio climático en una muestra de población adulta de nivel cultural medio. Serie Geográfica. Universidad de Alcalá de Henares., 1994, vol. 4, p. 131-136.

Correa, M. I. (2015). La Percepción Social del Cambio Climático. Cambio climático y representaciones sociales entre estudiantes de educación superior.

Chambers, R. (2009). Manual para el análisis de la capacidad y vulnerabilidad climática (en linea). CARE International. Consultado set 2012.

Evans, A., \& Steven, D. (2007). Climate change: the state of the debate. Center for International Cooperation, New York University.

Flores, R. C. (2015). Las representaciones sociales del cambio climático en estudiantes de educación secundaria. REXE-Revista de Estudios y Experiencias en Educación, 14(27), 15-32.

González, E. J., \& Maldonado, A. L. (2014). ¿ Qué piensan, dicen y hacen los jóvenes universitarios sobre el cambio climático? Un estudio de representaciones sociales. Educar en Revista, 35-55.

Hernández, R., Fernández, C., \& Baptista, P. (2010). Metodología de la investigación . México, DF: México: McGraw Hill.

IPCC. (2014). Cambio climático. Impactos, adaptación y vulnerabilidad. Resumen para responsables de políticas. Ginebra, Suiza. Contribución del Grupo de trabajo II al Quinto Informe de Evaluación del Grupo Intergubernamental de Organización Meteorológica Mundial.

Kaltenborn, B. P., Krange, O., \& Tangeland, T. (2017). Cultural resources and public trust shape attitudes toward climate change and preferred futures-A case study among the Norwegian public. Futures, 89, 1-13. doi: https://doi.org/10.1016/j.futures.2017.04.005

Leombruni, L. V. (2015). How you talk about climate change matters: A communication network perspective on epistemic skepticism and belief strength. Global Environmental Change, 35, 148-161. doi: http://doi.org/10.1016/j.gloenvcha.2015.08.006

Martínez, M. (2006). La investigación cualitativa (síntesis conceptual). Revista de investigación en psicología, 9(1), 123-146.

Moraes, D. S., \& Montes, I. G. (2011). Vulnerabilidad social, institucionalidad y percepciones sobre el cambio climático: un acercamiento al municipio de San Felipe, Costa de Yucatán. CIENCIA ergo-sum, 18(3), 249-263.

Moscovici, S. (1984). The Phenomenon of Social Representations. W: RM Farr, S. Moscovici (red.), Social Representations (s. 3-69): Cambridge: Cambridge University Press. 
Bio - grafía. Escritos sobre la Biología y su Enseñanza. ISSN 2027-1034

Edición Extraordinaria. p.p. 839 - 847

Memorias del IX Encuentro Nacional de Experiencias en Enseñanza de la Biología y la Educación Ambiental. IV Congreso Nacional de Investigación en Enseñanza de la Biología.

Muñoz, P., \& Muñoz, I. (2001). Intervención en la familia: estudio de casos. Modelos de investigación cualitativa en educación social y animación sociocultural, 221-254.

Pearce, W., Brown, B., Nerlich, B., \& Koteyko, N. (2015). Communicating climate change: conduits, content, and consensus. Wiley Interdisciplinary Reviews: Climate Change, 6(6), 613-626.

Retamal, M. R., Rojas, J., \& Parra, Ó. (2011). Percepción al cambio climático ya la gestión del agua: aportes de las estrategias metodológicas cualitativas para su comprensión. Ambiente \& Sociedade, 14(1), 175-194.

Rozell, D. (2017). Using population projections in climate change analysis. Climatic Change, 142(4), 1-9. doi: DOI 10.1007/s10584-017-1968-2

Serrano, G. P. (1994). Investigación cualitativa: retos e interrogantes. Métodos: La muralla.

Simons, H. (2011). El estudio de caso: Teoría y práctica: Ediciones Morata.

Stake, R. (2007). Investigación con estudio de caso (Ediciones Morata ed.). Madrid.

Vide, J. M. (2008). La nueva realidad del calentamiento global. Un decálogo del cambio climático. Scripta Nova: Revista Electrónica De Geografía y Ciencias Sociales, 12, 19. 